\title{
Modelling Ethylene-Hydrogen Jet Flames in the MILD Combustion Regime
}

\author{
M.J. Evans ${ }^{a}, \underline{\text { Z.F. Tian }}^{\mathrm{a}}{ }^{\text {and P.R. Medwell }}{ }^{\mathrm{a}}$ \\ ${ }^{a}$ University of Adelaide, Adelaide, South Australia, 5005 \\ Email: m.evans@adelaide.edu.au
}

\begin{abstract}
Moderate or Intense Low oxygen Dilution (MILD) combustion is a particular combustion regime which offers improved thermal efficiency and a reduction of nitrogen oxide $\left(\mathrm{NO}_{\mathrm{X}}\right)$ pollutants and soot. In this paper computational fluid dynamics (CFD) is employed to model turbulent jet flames issuing into a hot and diluted coflow stream, with a view to develop fundamental level understanding of the MILD combustion regime. Reynolds averaged Navier-Stokes (RANS) simulations are coupled with the Eddy Dissipation Concept (EDC) turbulence-chemistry model and the computational results are compared with experimental measurements. For the turbulent ethylene-hydrogen $\left(\mathrm{C}_{2} \mathrm{H}_{4} / \mathrm{H}_{2}\right)$ fuel jet, a modified $k-\varepsilon$ turbulence model, the standard Reynolds Stress Model (RSM), Shear Stress Transport (SST) model, $k-\omega$ models, and a modified RSM are considered. Results show that a mesh, constructed with 53610, primarily rectangular, elements with a characteristic length of $0.950 \mathrm{~mm}$, is sufficient to model the combustion processes in the MILD configuration of the JHC burner. The most accurate reacting flow field is predicted using the modified RSM, by adjustment of the factor $C_{1 \varepsilon}$ to 1.6 from the default 1.44 . The RSM is the most computationally expensive model, being an anisotropic extension of the standard $k-\varepsilon$ model, however the increased computational cost is small in comparison to the cost of solving the detailed, finite-rate chemistry required for modelling MILD combustion. The modified RSM is therefore deemed to be superior in this application in comparison to the other turbulence models investigated.
\end{abstract}

Keywords: Turbulence models, RANS, Combustion, MILD Combustion 


\section{INTRODUCTION}

The contemporary world is dependent on combustion. Moderate or Intense Low oxygen Dilution (MILD) combustion is a particular combustion regime which offers improved thermal efficiency and a reduction of nitrogen oxide $\left(\mathrm{NO}_{\mathrm{X}}\right)$ pollutants and soot (Cavaliere and de Joannon, 2004). MILD combustion is achieved by recirculating hot combustion products back into the flame front, creating a distributed reaction zone, which reduces pressure variations and lowers the peak flame temperature. The result is a reduction in fuel consumption and cleaner exhaust gases. MILD combustion has therefore been successfully employed in recirculating furnaces (Cavaliere and de Joannon, 2004).

To investigate the flame stabilisation mechanisms within the MILD combustion regime, the University of Adelaide (UoA) developed the Jet in Hot Coflow (JHC) burner, described by Medwell et al. (2008) and shown in Figure 1. The JHC burner consists of an insulated $\varnothing 4.6 \mathrm{~mm}$ central fuel jet which issues from a sufficiently long pipe to give a fully developed flow, within a coflow produced by a concentric $\varnothing 82 \mathrm{~mm}$ premixed flat-flame burner.

The experimental study of gaseous fuel mixtures in laboratory-scale MILD combustion burners (e.g. Dally et al., 2002; Medwell et al., 2008) is of interest for modelling, providing turbulent attached jet flames. Methane/hydrogen fuels have been modelled using the $k-\varepsilon$ turbulence model (Christo and Dally, 2005; Frassoldati et al., 2010; Mardani et al., 2010; Aminian et al., 2011), the Reynolds-stress model (RSM) (Frassoldati et al., 2010), the $k-\varepsilon$ model using renormalization group methods (RNG) (Christo and Dally, 2005; Aminian et al., 2011) and the realisable $k-\varepsilon$ model (Christo and Dally, 2005; Aminian et al., 2011). A recent study of ethylene-

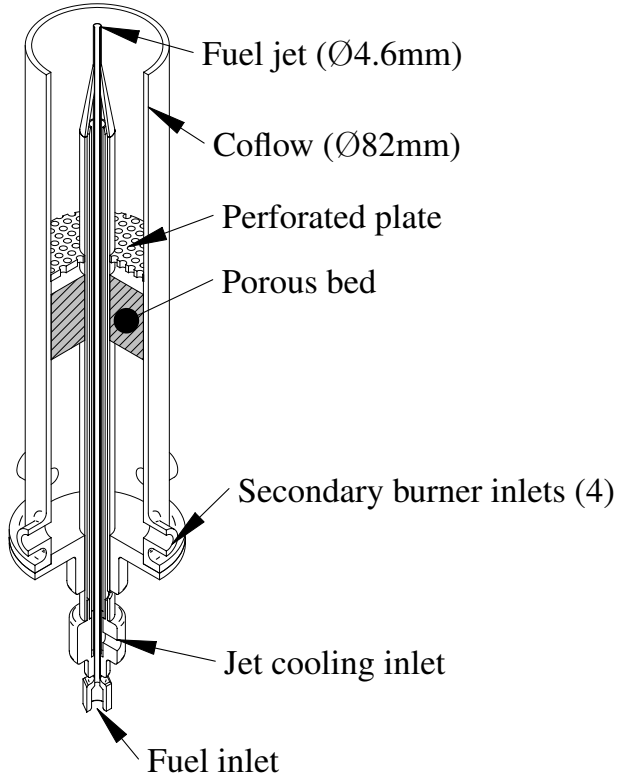

Figure 1. Schematic of the JHC burner used for MILD combustion experiments (Medwell et al., 2008).

based fuel mixtures reported good agreement between experiment and both modified $k$ - $\varepsilon$ model and standard RSM (Shabanian et al., 2013). The RSM is, notably, an extension of the $k-\varepsilon$ model without the assumption of isotropic eddy viscosity which assumes that the shear stress in a fluid is proportional to the strain rate. This paper investigates the effects of the $k-\omega$ (where $\omega$ is the specific dissipation rate) and shear-stress transport (SST) models on the accuracy of modelling ethylene/hydrogen $\left(\mathrm{C}_{2} \mathrm{H}_{4} / \mathrm{H}_{2}\right)$ flames, in addition to the effect of using the modified the RSM in accordance with the modified $k-\varepsilon$ model as done by Dally et al. (1998).

Computational fluid dynamics (CFD) may employ different turbulence models to approximate solutions of the Reynolds averaged Navier-Stokes (RANS) equations and chemical reactions to simulate combustion processes. One approach of dealing with finite-rate turbulence-chemistry interactions is with the Eddy Dissipation Concept (EDC) model (Magnussen, 1981). The EDC model was found by Christo and Dally (2005) to model combustion of methane in the JHC burner with reasonable accuracy in regions without local extinction or apparent lift-off. The suitability of this turbulence-chemistry model was further confirmed with a different burner, also operating in the MILD regime (Galletti et al., 2007). Current EDC models of the JHC burner, however, still exhibit inaccuracies in modelling $\mathrm{C}_{2} \mathrm{H}_{4} / \mathrm{H}_{2}$ flames in a $9 \% \mathrm{~mol} / \mathrm{mol} \mathrm{O}_{2}$ coflow in the JHC and do not accurately predict temperature and molecular species distributions measured experimentally across all fuel and coflow cases. Accurate computational modelling of the JHC would complement and extend experimental studies and the understanding of fundamental mechanics in this combustion regime.

\section{MODEL DEVELOPMENT}

The CFD package chosen to be used was ANSYS FLUENT 14.0, in conjunction with the GRI-Mech 3.0 chemical kinetics mechanism, which has previously been shown to successful at modelling MILD combustion (e.g. Christo and Dally, 2005; Frassoldati et al., 2010; Shabanian et al., 2013).

\subsection{Computational Domain}


Geometry. The computational domain for the JHC burner was chosen to be a two-dimensional rectangular region downstream of the jet plane exit. The geometry for this study was based on the computational domains of Frassoldati et al. (2009) and Shabanian et al. (2013). The 400mm ( $~ 85$ jet diameters) downstream extent of this domain, shown in Figure 2, captures the entire MILD combustion regime, and sufficient flame length such that flame interaction with the domain exit will not influence the region of interest. The full length of the flame extends beyond the domain, however combustion in the downstream part of the flame is not in the MILD regime (because it is outside the potential core of the coflow) and thus not controlled.

The soot-free nature of MILD combustion also implies that radiation heat exchange between the region near the jet exit plane and the downstream flame is very low. The low radiative heat exchange, consequently, suggests little dependence of the result on the choice of radiation model. The virtual independence of radiation model on the region near the jet exit has been confirmed by previous studies (e.g Frassoldati et al., 2010; Shabanian et al., 2013, Supplement Material), with radiation ignored (e.g Mardani et al., 2010) or included (e.g. Aminian et al., 2011) by others. From these conclusions, the 'P1' radiation model, the most basic radiation model commonly available, will be implemented to retain accuracy away from the jet at a minimal computational cost.

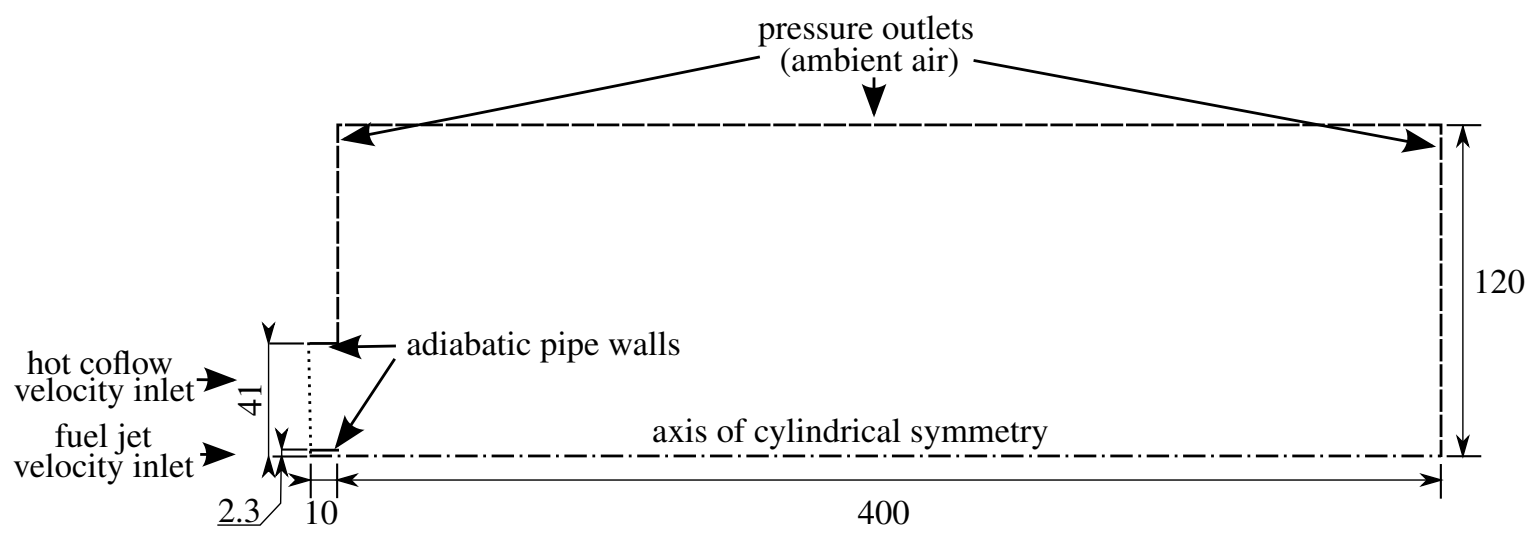

Figure 2. Geometry of computational domain and boundary conditions for combustion model, dimensions in $\mathrm{mm}$. Note that the jet and coflow inlets represent 4.6 and $82 \mathrm{~mm}$ diameters respectively, separated by a $0.2 \mathrm{~mm}$ thick wall. Pressure Outlets (- $)$, Axis of Cylindrical Symmetry (- - -), Adiabatic Pipe Walls (-) and Velocity Inlets $(\cdots)$.

Boundary Conditions. The boundaries of the domain were a combination of walls, pressure outlets, velocity inlets and the axis of cylindrical symmetry through the centre of the coaxial jets. The domain, with labelled boundary conditions, is shown as Figure 2. Adiabatic, wall boundaries were used to describe the pipe walls in the JHC burner, imposing no-slip (zero fluid velocity) conditions on the wall surface. Pressure outlets specify the ambient surrounds to be simplified air, with $21 \% \mathrm{O}_{2}$ and $79 \% \mathrm{~N}_{2} \mathrm{~mol} / \mathrm{mol}$, at zero gauge pressure and a temperature of $300 \mathrm{~K}$. In addition to being an outlet to allow fluids to leave the domain, pressure outlets allow external air to flow into low pressure regions of the domain, conserving mass in the domain during simulations. The primary inlets in this domain are the fuel and coflow velocity inlets. Fluids enter the domain through these boundaries with set compositions, pressures, temperatures, flow rates and turbulence characteristics chosen to best represent the physical characteristics of the experimental conditions. The fuel stream, entering the computational domain perpendicular to the velocity inlet boundary at $30.6 \mathrm{~m} / \mathrm{s}, 305 \mathrm{~K}$ and zero gauge pressure was set to be $50 \% \mathrm{C}_{2} \mathrm{H}_{4}$ and $50 \% \mathrm{~mol} / \mathrm{mol} \mathrm{H}_{2}$, and the $2.3 \mathrm{~m} / \mathrm{s}, 1100 \mathrm{~K}$ coflow was taken as $9 \% \mathrm{O}_{2}, 3 \% \mathrm{CO}_{2}$, $78 \% \mathrm{~N}_{2}$ and $10 \% \mathrm{H}_{2} \mathrm{O} \mathrm{mol} / \mathrm{mol}$ (Medwell et al., 2008). Inlet velocity profiles into the domain were assumed to take a 'top-hat' form, though zero at pipe walls, due to a lack of information in the literature on the effects of inlet profiles on modelling MILD combustion.

\subsection{Mesh Generation}

In this study, three unique meshes were constructed using a rectangular approach, and defined by the representative mesh size, $h$ as the square-root of the average element area, or the average edge length of the rectangular element mesh, as defined by Celik et al. (2008). The ratio $h$ between any two meshes met the requirement that $r_{12}=h_{1} / h_{2} \geq 1.3$ where $h_{1}$ represents the coarser of the two meshes. Three distinguishable meshes for the reacting flow cases, numbered 1-3 featured 53610, 149060 and 251916 elements respectively, with 
$h_{1}=0.950 \mathrm{~mm}, h_{2}=0.570 \mathrm{~mm}$ and $h_{3}=0.438 \mathrm{~mm}$. The meshes were required to be sufficiently fine to capture details of the flow field in mixing regions where combustion was anticipated. These needed to accurately estimate the fluid behaviour in each element and estimate the local parameters and species concentrations required for the EDC combustion model. Mesh independence studies observed the effects of the choice of mesh on the resulting flow field, to ensure the results were not numerical artefacts of the mesh. No lower limit was placed on the smallest element size, with truncation errors reduced by implementing the double-precision solver available in FLUENT 14.0.

\subsection{Turbulence and Chemistry Models}

The modified EDC model of (Shabanian et al., 2013) was chosen for use with all turbulent models in light of its reported success. The results of $k-\varepsilon$ turbulence model were also used for the normalisation of both hydroxyl radical $(\mathrm{OH})$ and formaldehyde $\left(\mathrm{CH}_{2} \mathrm{O}\right)$ concentrations at the $35 \mathrm{~mm}$ downstream location where experimental measurements are available.

The modified $k$ - $\varepsilon$ turbulence model is adjusted from the standard model by changing the common default value of $C_{1 \varepsilon}$, in equations for scalar dissipation rate, from 1.44 to 1.6 for 2D axisymmetric flows (Dally et al., 1998). As the anisotropic extension of the eddy viscosity, $k-\varepsilon$ model leads to the RSM, this modification is available for RSM simulations and the accuracy of this modification has been previously verified for non-reacting jets (Dally et al., 1998). Therefore other turbulence models considered were the standard RSM, a modified RSM with the same choice of $C_{1 \varepsilon}=1.6$, the $k-\omega$ and shear-stress transport (SST) models, with FLUENT 14.0 default parameters with the exception of the $C_{1 \varepsilon}$ modification.

\subsection{Solution Convergence}

Numerical results on each mesh were solved iteratively in FLUENT, using second order finite difference methods, with variable imbalance residuals calculated after each iteration. The root-mean-squared value of the residuals for each variable were subsequently scaled by the difference between the maximum and minimum value of the variable of the domain. The target value for scaled residuals was $10^{-3}$ for continuity and $10^{-5}$ for momentum, energy, radiation and species concentration. In addition to monitoring the scaled residuals over the computational domain, the iterative history of values of interest within the solution was used as a measure of convergence. These values of interest included temperatures and selected species concentrations at different downstream locations and the peak values of temperature, $\mathrm{OH}$ and $\mathrm{CH}_{2} \mathrm{O}$ in the plane $35 \mathrm{~mm}$ downstream from the jet exit, shown later in Figure 4. The decrease of scaled residuals to below a target value, and the iterative convergence of values of interest were used to determine the convergence of a final solution. In some cases, where the residuals levelled off after reaching the same order of magnitude as the convergence criteria, iterative convergence was assumed in cases where the values of interest were invariant with iterations. This was particularly noted for the solution obtained in the RSM turbulence case, which is known to suffer convergence difficulties in some cases.

\section{RESULTS}

\subsection{Non-Reacting Verification}

Mesh independence of a non-reacting jet into quiescent (still) air was studied on four meshes, including meshes 1-3 and a fourth, coarser mesh with 29292 elements and $h_{0}=1.29 \mathrm{~mm}$ - which suffered convergence difficulties and resulted in a noticeably different flow field. These used the previously verified, modified $k-\varepsilon$ model (Dally et al., 1998; Christo and Dally, 2005). Away from the jet exit, these exhibited centreline velocity and jet species concentration decays inversely proportional to axial distance and the radial profiles of both velocity and jet species grew linearly with axial distance, termed downstream 'self-similarity', as seen experimentally (Chen and Rodi, 1980). Good agreement was, additionally, seen between the standard and modified RSMs, predicting self-similarity for axial distances up to $\sim 60$ jet diameters downstream. The species concentration profile expansion rate predicted by the two RSMs was in better agreement to empirical predictions, however, the modified $k-\varepsilon$ model better predicted concentration decay along the centreline and the velocity field.

\subsection{Reacting Models of $\mathrm{C}_{2} \mathrm{H}_{4} / \mathrm{H}_{2}$ Combusiton in the JHC}

Visual comparisons of the $\mathrm{CH}$ species concentration contour and the volumetric average of $\mathrm{CH}$ concentration using the $k-\varepsilon$ model are shown alongside experimental measurements in Figure 3. Concentration contours quantitatively describe the concentration of the $\mathrm{CH}$ radical (which is closely correlated to the $\mathrm{CH}^{*}$ radical 
whose chemiluminescence is responsible for the characteristic 'blue flame') in a section-plane of the $\mathrm{C}_{2} \mathrm{H}_{4} / \mathrm{H}_{2}$ axisymmetric flame. The visual appearance of a real flame is, however, the sum of light emission through the entire thickness of the flame. The volume-averaged $\mathrm{CH}$ profile, right-most in Figure 3, shows how the $\mathrm{CH}$ concentration varies throughout the flame simulated using the $k-\varepsilon$ model, compared to the observed chemiluminescence of $\mathrm{CH}^{*}$.
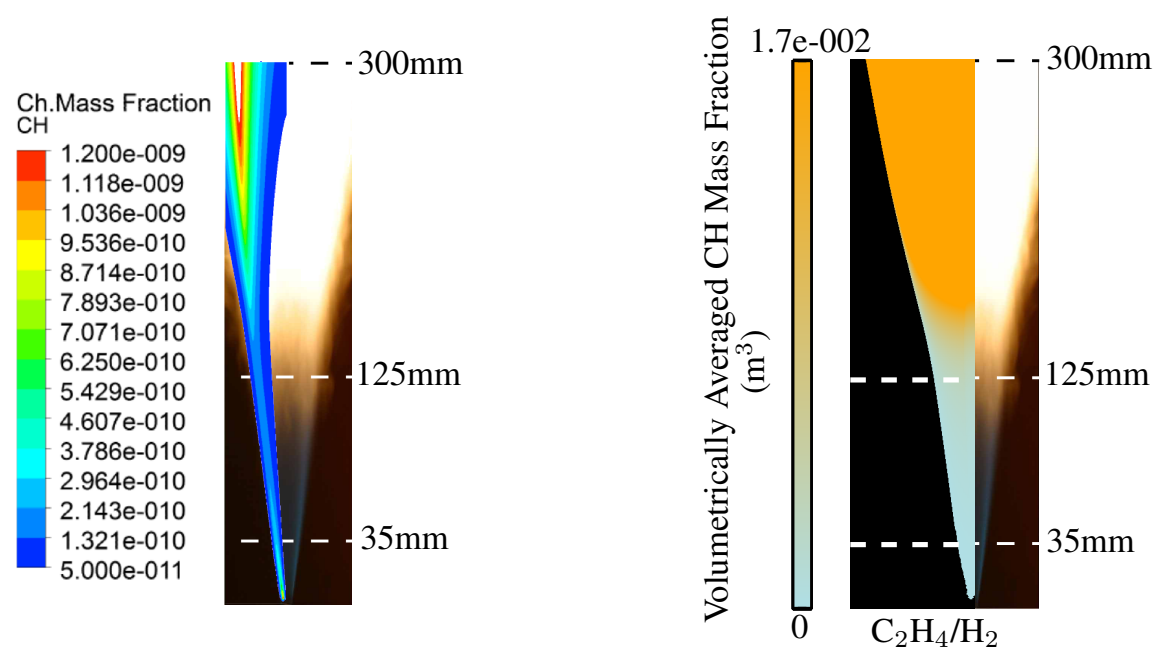

Figure 3. Visual comparisons of $\mathrm{CH}$ distribution in modified $k-\varepsilon$ results (left-hand side of each image) and experimental (right-hand side) $\mathrm{C}_{2} \mathrm{H}_{4} / \mathrm{H}_{2}$ fuel flames in a $9 \% \mathrm{~mol} / \mathrm{mol} \mathrm{O} \mathrm{O}_{2}$ coflow.

Figure 4 shows a comparison of the modelled results of temperature, normalised $\mathrm{OH}$ and $\mathrm{CH}_{2} \mathrm{O}$ concentrations against the experimental data. These plots show both the mesh independence study of the modified $k$ - $\varepsilon$ model, and the comparison of the other turbulence models investigated in this study.

\section{DISCUSSION}

Direct comparison of the visual measurements and volumetrically averaged $\mathrm{CH}$ contours show that the modified $k$ - $\varepsilon$ turbulence model and the modified EDC combustion model of Shabanian et al. (2013) reasonably reproduces $\mathrm{CH}$ concentrations of $\mathrm{C}_{2} \mathrm{H}_{4} / \mathrm{H}_{2}$ fuel flames in the JHC in a $9 \% \mathrm{~mol} / \mathrm{mol} \mathrm{O}_{2}$ coflow. The results in Figure 4 show that the results of the modified $k-\varepsilon$ turbulence model are independent of the mesh size over the range of meshes $1-3$, implying that the 53610 element mesh $_{1}$ should be adequate for use further investigations using this model. The good convergence and simple geometry of the case, indicate that mesh is likely to be sufficient for similar RANS models, such as the RSM, $k-\omega$ and SST models.

The modified $k-\varepsilon$ model notably over-predicts the maximum temperature and radial locations of the maximum temperature and $\mathrm{OH}$ species concentration in the $35 \mathrm{~mm}$ downstream plane. Only the $k$ - $\omega$ model drastically over-predicts the radial location of peak temperature, $\mathrm{OH}$ and the shape of their distributions. The modified $k$ - $\varepsilon$ model does, however, accurately predict the location of the local maximum $\mathrm{CH}_{2} \mathrm{O}$. The experimental peak centred about the domain axis is suspected to be a measurement anomaly or interference and is therefore not represented in any of the numerical models and hence ignored. Reasonable agreement in predicting the maximum $\mathrm{CH}_{2} \mathrm{O}$ location is seen for all models, however the modified RSM appears to show both the most accurate location of this peak and, best matches the slope of the peak. Despite the standard RSM and SST models best predicting the maximum temperature, the modified RSM best locates the maximum temperature and $\mathrm{OH}$ concentrations radially. The shape of the standard and modified RSM data are quite similar, albeit shifted radially in the plots in Figure 4. This increased accuracy of the RSM with $C_{1 \varepsilon}=1.6$, suggests either that the boundary conditions of the model - following those used in previous studies (e.g. Christo and Dally, 2005; Frassoldati et al., 2010; Mardani et al., 2010; Aminian et al., 2011; Shabanian et al., 2013) - lack sufficient physical detail or that the RSM should be subject to the same modification as its simplified counterpart, the $k-\varepsilon$ model in order to better describe MILD combustion.

The visual comparison in Figure 3 demonstrates that combustion occurs, as expected, immediately near the jet exit indicating that the inlet conditions provide sufficient mixing for turbulent combustion. These images appear to show a very small predicted flame lift-off from the jet, not present in the optical measurements. This 

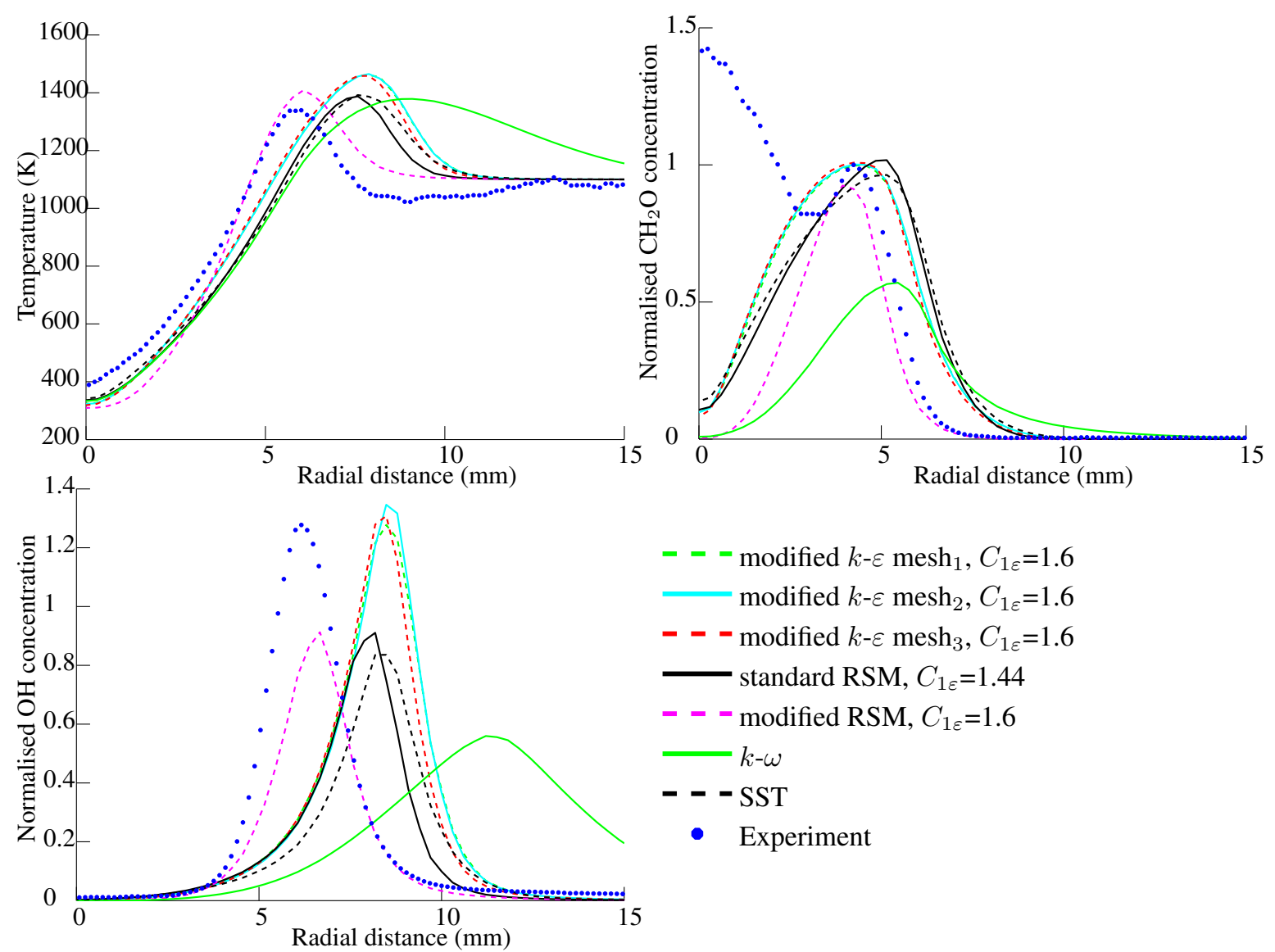

$$
\begin{aligned}
& \text { - - " modified } k-\varepsilon \operatorname{mesh}_{1}, C_{1 \varepsilon}=1.6 \\
& \text { modified } k-\varepsilon \operatorname{mesh}_{2}, C_{1 \varepsilon}=1.6 \\
& \text { - - - modified } k \text { - } \varepsilon \operatorname{mesh}_{3}, C_{1 \varepsilon}=1.6 \\
& \text { - standard RSM, } C_{1 \varepsilon}=1.44 \\
& \text { - - - modified RSM, } C_{1 \varepsilon}=1.6 \\
& -k-\omega \\
& \text { - - - SST }
\end{aligned}
$$

Figure 4. Grid convergence of the modified $k-\varepsilon$ model and the comparison of results using different turbulence models against experimental data measured at $35 \mathrm{~mm}$ downstream of the $\mathrm{JHC}$ jet exit plane for $\mathrm{C}_{2} \mathrm{H}_{4} / \mathrm{H}_{2}$ fuel in a $9 \% \mathrm{~mol} / \mathrm{mol} \mathrm{O}_{2}$ coflow (Medwell et al., 2008). Flow field solved on $\mathrm{mesh}_{1}$ unless stated otherwise, using the modified EDC combustion model and a modified GRI-Mech 3.0 mechanism.

may be attributed to high fluid strain rates in mixing near the jet and coflow exits, due to the fuel velocity being an order of magnitude greater than the coflow velocity. More accurate boundary conditions would likely reduce, or eliminate, this gap between the jet exit and initial combustion, which would likely increase the temperature and size of the flame at the $35 \mathrm{~mm}$. The flame predicted by all but the modified RSM model is, however, already larger than the experimentally measured flame in the radial extent, and as such changing the boundary conditions is not likely to offer any significant improvement in predictions $35 \mathrm{~mm}$ downstream of the jet exit.

The non-reacting comparison of empirical predictions, modified $k-\varepsilon$ model, the standard and modified RSMs demonstrated how both RSMs better agree with the species concentration profile expansion rate downstream of the jet exit. This improvement, in conjunction with the findings of Dally et al. (1998) that the modified RSM outperforms the standard RSM, standard $k-\varepsilon$ and - to a lesser extent - the modified $k-\varepsilon$ models for 2D axisymmetric jets, indicates that the modified RSM provides a more accurate description of the flow field and, hence, temperature and species concentrations. The improvement in accuracy due to modification of $C_{1 \varepsilon}$ is a consequence of the increased turbulent eddy dissipation rate and, accordingly, decreased turbulence intensity and hence decreased jet spreading downstream of the jet exit. The use of this model was not recommended by Dally et al. (1998) at the time, due to the inherently increased computational expense of the anisotropic RSM compared the simpler isotropic $k-\varepsilon$ model. This increased computational expense is small in comparison to the resources required by the detailed chemical kinetics used for modelling the MILD combustion of $\mathrm{C}_{2} \mathrm{H}_{4}$-based fuels, and the use of the modified RSM - which may be initiated from a partially converged modified $k-\varepsilon$ solution - is easily justified by the increased accuracy, and recommended for this fuel and coflow case.

\section{CONCLUSIONS}

The JHC enables controlled MILD combustion. A $50 \% \mathrm{C}_{2} \mathrm{H}_{4}$ and $50 \% \mathrm{~mol} / \mathrm{mol} \mathrm{H}_{2}$ fuel jet, in a $1100 \mathrm{~K}, 9 \%$ $\mathrm{O}_{2}$ coflow was modelled using a modified $k-\varepsilon$ model, standard $k-\omega$ and SST models, a standard RSM and an 
RSM modified in the same manner as the modified $k-\varepsilon$ model. All turbulence models except the $k$ - $\omega$ model demonstrated reasonable agreement with experimental data from $35 \mathrm{~mm}$ downstream of the jet exit, used in conjunction with detailed, finite-rate chemical kinetics. The most accurate results are found using the RSM with the modification $C_{1 \varepsilon}=1.6$. The RSM is the most sophisticated turbulence model used in this study, and the modification is identical to that used for $2 \mathrm{D}$ axisymmetric models in the related $k-\varepsilon$ model. This modified RSM gives more accurate results in this simulation, at only a slightly greater computational expense than the widely used modified $k-\varepsilon$ model.

\section{REFERENCES}

Aminian, J., C. Galletti, S. Shahhosseini, and L. Tognotti (2011). Key modeling issues in prediction of minor species in diluted-preheated combustion conditions. Applied Thermal Engineering 31(16), 3287-3300.

Cavaliere, A. and M. de Joannon (2004). Mild combustion. Progress in Energy and Combustion Science 30(4), 329-366.

Celik, I. B., U. Ghia, P. J. Roache, and Christopher (2008, Jul). Procedure for estimation and reporting of uncertainty due to discretization in CFD applications. Journal of fluids Engineering-Transactions of the ASME 130(7).

Chen, C. J. and W. Rodi (1980). Vertical turbulent buoyant jets : a review of experimental data, Volume 4 of $H M T$, the science applications of heat and mass transfer. Pergamon Press Ltd.

Christo, F. C. and B. B. Dally (2005). Modeling turbulent reacting jets issuing into a hot and diluted coflow. Combustion and Flame 142(1-2), 117-129.

Dally, B., A. Karpetis, and R. Barlow (2002). Structure of turbulent non-premixed jet flames in a diluted hot coflow. In Proceedings of the Combustion Institute, Volume 29, pp. 1147-1154.

Dally, B. B., D. F. Fletcher, and A. R. Masri (1998). Flow and mixing fields of turbulent bluff-body jets and flames. Combustion Theory and Modelling 2(2), 193-219.

Frassoldati, A., A. Cuoci, T. Faravelli, E. Ranzi, S. Colantuoni, P. D. Martino, and G. Cinque (2009). Experimental and modeling study of a low $\mathrm{NO}_{x}$ combustor for aero-engine turbofan. Combustion Science and Technology 181(3), 483-495.

Frassoldati, A., P. Sharma, A. Cuoci, T. Faravelli, and E. Ranzi (2010). Kinetic and fluid dynamics modeling of methane/hydrogen jet flames in diluted coflow. Applied Thermal Engineering 30(4), 376-383.

Galletti, C., A. Parente, and L. Tognotti (2007). Numerical and experimental investigation of a mild combustion burner. Combustion and Flame 151, 649-664.

Magnussen, B. F. (1981). On the structure of turbulence and a generalized eddy dissipation concept for chemical reaction in turbulent flow. In 19th AIAA Aerospace Meeting, St. Louis, USA.

Mardani, A., S. Tabejamaat, and M. Ghamari (2010). Numerical study of influence of molecular diffusion in the mild combustion regime. Combustion Theory and Modelling 14(5), 747-774.

Medwell, P. R., P. A. M. Kalt, and B. B. Dally (2008). Imaging of diluted turbulent ethylene flames stabilized on a jet in hot coflow (JHC) burner. Combustion and Flame 152, 100-113.

Shabanian, S. R., P. R. Medwell, M. Rahimi, A. Frassoldati, and A. Cuoci (2013). Kinetic and fluid dynamic modeling of ethylene jet flames in diluted and heated oxidant stream combustion conditions. Applied Thermal Engineering 52, 538-554. 